\title{
Two-speed phacoemulsification for soft cataracts using optimized parameters and procedure step toolbar with the CENTURIONVision System and Balanced Tip
}

\author{
This article was published in the following Dove Press journal: \\ Clinical Ophthalmology \\ 27 August 2015 \\ Number of times this article has been viewed
}

James A Davison

Wolfe Eye Clinic, Marshalltown, IA, USA
Correspondence: James A Davison Wolfe Eye Clinic, 309 East Church Street, Marshalltown, IA 50I58, USA Email jdavison@wolfeclinic.com
Purpose: To present a cause of posterior capsule aspiration and a technique using optimized parameters to prevent it from happening when operating soft cataracts.

Patients and methods: A prospective list of posterior capsule aspiration cases was kept over 4,062 consecutive cases operated with the Alcon CENTURION machine and Balanced Tip. Video analysis of one case of posterior capsule aspiration was accomplished. A surgical technique was developed using empirically derived machine parameters and customized setting-selection procedure step toolbar to reduce the pace of aspiration of soft nuclear quadrants in order to prevent capsule aspiration.

Results: Two cases out of 3,238 experienced posterior capsule aspiration before use of the soft quadrant technique. Video analysis showed an attractive vortex effect with capsule aspiration occurring in $1 / 5$ of a second. A soft quadrant removal setting was empirically derived which had a slower pace and seemed more controlled with no capsule aspiration occurring in the subsequent 824 cases. The setting featured simultaneous linear control from zero to preset maximums for: aspiration flow, $20 \mathrm{~mL} / \mathrm{min}$; and vacuum, $400 \mathrm{mmHg}$, with the addition of torsional tip amplitude up to $20 \%$ after the fluidic maximums were achieved. A new setting selection procedure step toolbar was created to increase intraoperative flexibility by providing instantaneous shifting between the soft and normal settings.

Conclusion: A technique incorporating a reduced pace for soft quadrant acquisition and aspiration can be accomplished through the use of a dedicated setting of integrated machine parameters. Toolbar placement of the procedure button next to the normal setting procedure button provides the opportunity to instantaneously alternate between the two settings. Simultaneous surgeon control over vacuum, aspiration flow, and torsional tip motion may make removal of soft nuclear quadrants more efficient and safer.

Keywords: complication, divide and conquer, INFINITI Vision System, posterior capsule CENTURION Vision System, cataract surgery

\section{Introduction}

Phacoemulsification of hard cataracts receives much attention but many times it is the softer ones which can present challenges and require special techniques. ${ }^{1,2}$ The direct jackhammer effect of the tip's impact on hard lens material emulsifies it turning it into particulate dust within a stream of balanced salt solution (BSS) (Alcon Laboratories, Inc., Fort Worth, TX, USA). Cavitation is thought to be a secondary and perhaps negligible effect. ${ }^{3}$ In softer material, a relatively formed nuclear gel is drawn through the shaft by vacuum and the flow created by pressure differential. The movement is accelerated as the gel deforms and is mixed with BSS, changing its viscosity because 
of increasing tip motion. Modern machines are capable of well-controlled high vacuum and flow, short maximum vacuum rise times, and low postocclusion break surge values. ${ }^{4,5}$ Those fluidic features combined with linear control of angled tips using longitudinal or torsional movement ${ }^{6-8}$ or a mix of them $^{9}$ make acquisition and initial emulsification of nuclear quadrants almost immediate, leading to very efficient surgery. Surgeons usually feel that they must dial in parameter values in the quadrant removal setting which optimize their techniques over a normal range of cataract hardness. But the use of those parameters may leave the capsules of soft lenses vulnerable to inadvertent aspiration. ${ }^{10}$

In this paper, I analyze slow-motion video of a case performed by our surgical team which shows an almost instantaneous attraction force reaching through the homogeneous nuclear gel of a soft cataract during phacoemulsification with the Alcon CENTURION machine and Balanced Tip. This force causes inadvertent posterior capsule aspiration. I present the evolution of a separate slow aspiration machine setting for the CENTURION to be used on soft cataracts and its location on the procedure step toolbar so that shifting between it and the normal setting can be convenient and immediate. I also present a technique for soft cataract removal and its results with respect to posterior capsule aspiration over a series of cases. The technique utilizes an oblique tip orientation and the use of the slow speed setting comprised of empirically derived parameters for vacuum, aspiration flow, and torsional tip movement. Simultaneous surgeon control of all three of these parameters seems to make acquisition and removal of soft nuclear fragments easier and safer.

\section{Materials and methods}

\section{Background surgical technique and initial CENTURION/Balanced Tip experience}

We started our conversion from the INFINITI phacoemulsification machines and Partial Kelman Tips to the CENTURION machines and Balanced Tips (all Alcon Laboratories, Inc.) in July 2013. We continued to use our modification of the divide and conquer technique using topical/intracameral anesthesia with $2.4 \mathrm{~mm}$ near-clear temporal main and left-handed accessory paracentesis corneal incisions $(2.4 \mathrm{~mm}$ bi-beveled knife and $22.5^{\circ}$ paracentesis knife, both from Alcon Laboratories, Inc.). ${ }^{11}$ We also continued to use the longitudinal tip motion for sculpting and the torsional with interjected vacuum triggered longitudinal pulses (Intelligent Phaco) for quadrant removal. The unique feature of the technique is that after deep sculpting and tearing the posterior nuclear plate into four quadrants using countertraction from a cyclodialysis spatula $(0.5$ modified to
$0.35 \mathrm{~mm}$ SP765852; Bausch \& Lomb Incorporated, Bridgewater, NJ, USA), the deep and central nuclear quarters are further debulked in situ with continued low vacuum and flow. This additional sculpting converts the quadrants into thinner relatively two-dimensional nuclear plates. After that, the high vacuum quadrant aspiration phase of surgery occurs within the nuclear bowl which is comprised of the thin plates. Most of quadrant removal activity occurs within the confines of the intracapsular space well away from the corneal endothelium.

It was immediately apparent that the CENTURION and Balanced Tip's performance would be a substantial change from the INFINITI and Partial Kelman's. Not only were the fluidics smoother with almost no iris movement or pupil reduction, but the cutting efficiency of the tip was extraordinary. Nuclear followability ${ }^{8}$ seemed to be substantially improved. That is, once material was acquired, it seemed to be adherent to the tip held tumbling over the aperture as it disappeared. Aspiration of material seemed much faster at all levels of nuclear hardness but seemed almost immediate in softer cataracts creating a concern when attracting them from their position in the periphery for centralized aspiration. Both tips feature a $150 \mu \mathrm{m}$ aperture (aspiration bypass system $[A B S]$ ) in their shaft to even out vacuum excursion extremes and prevent absolute loss of flow if the tip aperture were to be completely occluded. The aperture results in a $12 \%$ reduction in attained maximum vacuum. ${ }^{12}$

Because of the CENTURION's quick responsiveness, we felt it was necessary to reduce the values of the torsional tip movement, vacuum, and flow parameters that we had used with the INFINITI to achieve a similar paced quadrant removal experience. We ultimately derived and used the values which comprise our current quadrant removal setting (Figure 1). With a maximum vacuum of $400 \mathrm{mmHg}$ used for both machines, we would sometimes manually reduce the fixed aspiration flow rate when things seem to happen too quickly during quadrant removal of soft cataracts (INFINITI 35 to $25 \mathrm{~mL} / \mathrm{min}$ and CENTURION 24 to $20 \mathrm{~mL} / \mathrm{min}$ ).

\section{Posterior capsule aspiration cases and videography}

Four thousand and sixty-two consecutive CENTURION/ Balanced Tip cases were operated by a single surgeon (JAD) from July 2013 through June 2015. We kept a prospective list of inadvertent posterior capsule aspiration and found that two cases experienced that complication. A video recording of the first case was analyzed to study the capsule aspiration event. Because of those cases, we made machine programming adjustments after each. 


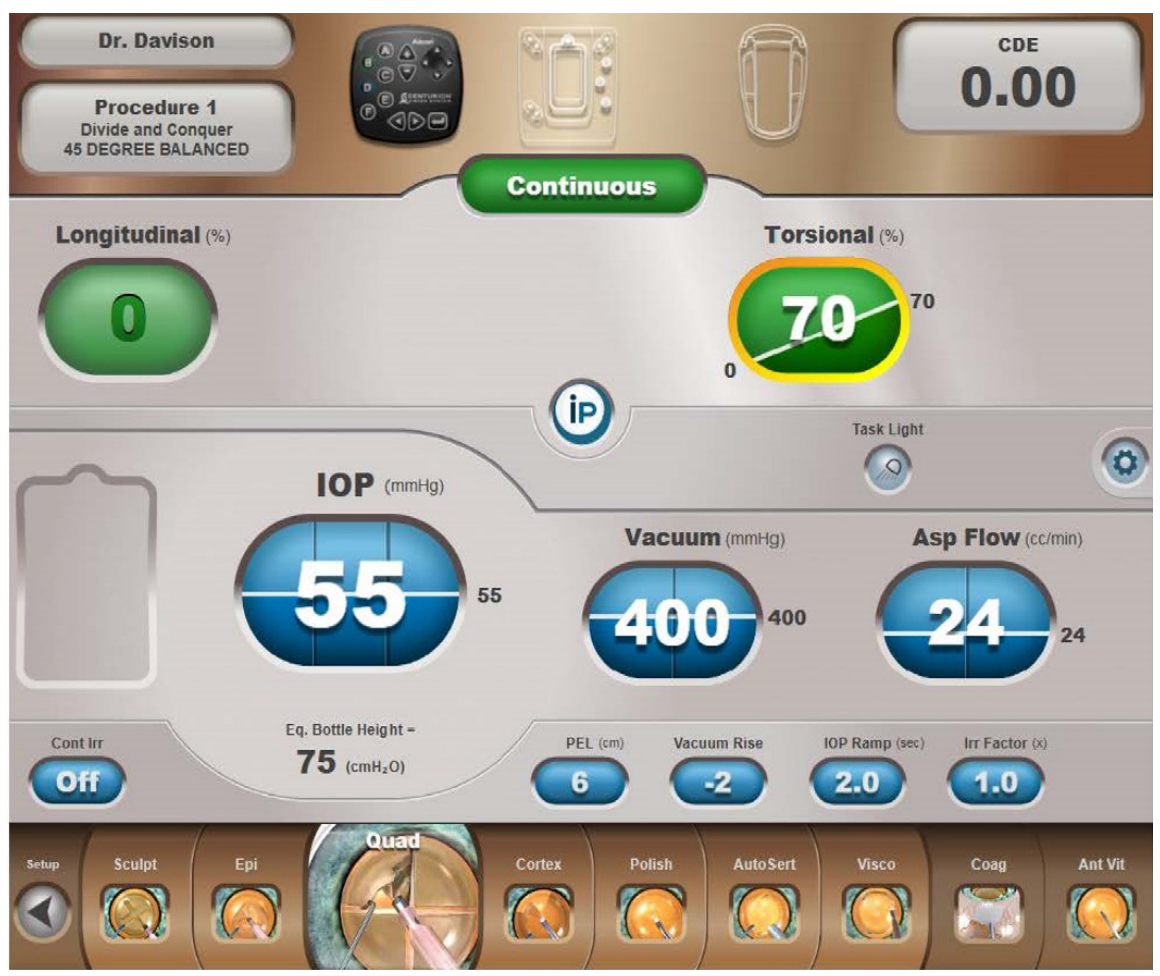

Figure I The machine panel display of the Quadrant Removal Setting (Quadrant Removal Step Button enlarged) in the procedure step toolbar shows a -2 vacuum rise with linear control (angled line) of torsional amplitude.

Notes: Vacuum and aspiration flow parameters are fixed (horizontal line) with moderate value maximums for foot position 2 (left side of vertical line) and 3 (right side of vertical line). A target IOP of $55 \mathrm{mmHg}$ is the equivalent of a gravity feed bottle height of $75 \mathrm{~cm}$ and has been selected for all three foot positions. The iP (Intelligent Phaco) feature is engaged with $10 \mathrm{msec}$ longitudinal pulse duration, $95 \%$ vacuum threshold, and 1.0 longitudinal/torsional ratio.

Abbreviation: IOP, intraocular pressure.

\section{Results}

\section{Posterior capsule aspiration cases, videography analysis, and setting adjustments}

The first case was a 61-year-old female operated in December 2013 after an initial 227 cases operated with the CENTURION. Her lens soft with respective LOCS III values for nuclear opalescence (NO) and nuclear color (NC) of 3.4 and 3.2 and we had intended to implant an Alcon 20.5 D SN60WF IOL. Even though a hole was aspirated in the posterior capsule during attempted removal of the first quadrant, the anterior hyaloid membrane remained intact and no vitreous was encountered. Employing air to maintain surface tension and pressure along the membrane and then Viscoat to replace the air, we were able to implant a three-piece posterior chamber IOL (Alcon 20.5 D MN60AC) with the haptics in the ciliary sulcus and the optic captured within the capsulorhexis border. Recovery was uneventful with recovery of $20 / 20$ vision with plano $-1.00 \times 068$ manifest refraction.

Video freeze frames from standard slow motion 30 frames/ second video (Figure 2) show the posterior capsule aspiration event. Analysis of the video showed that the posterior capsule was aspirated because of a distant vortex effect of vacuum and flow while the phacoemulsification tip was active. This attractive effect projected distant to the physical border of the phacoemulsification tip itself. Even though the tip was at approximately $1.5 \mathrm{~mm}$ from the posterior capsule, a column of tissue comprised of soft nucleus, cortex, and posterior capsule was drawn into the tip's aperture. After the small button of posterior capsule was aspirated, tension from the lens zonule retracted the remaining capsule and adjacent lens material back into their normal positions. In this case, enough fluid separated the anterior hyaloid membrane from the posterior capsule so that no vitreous was aspirated (Figure 3). The entire event took 0.3 seconds.

Because of that case, we derived a separate menu of settings to be used in soft cataract cases identified by LOCS III NO or NC values of 3.5 or less. In each instance of soft cataract, that soft cataract menu was engaged before the start of the case. The difference in parameter values during removal of soft quadrants was a reduction in torsional amplitude from linear with up to $70 \%$ of maximum available to linear with up to $20 \%$ available and a reduction in Aspiration Flow from fixed 24 to fixed $20 \mathrm{~mL} / \mathrm{min}$. Vacuum remained fixed at $400 \mathrm{mmHg}$ with a -2 Vacuum Rise. While seemingly 

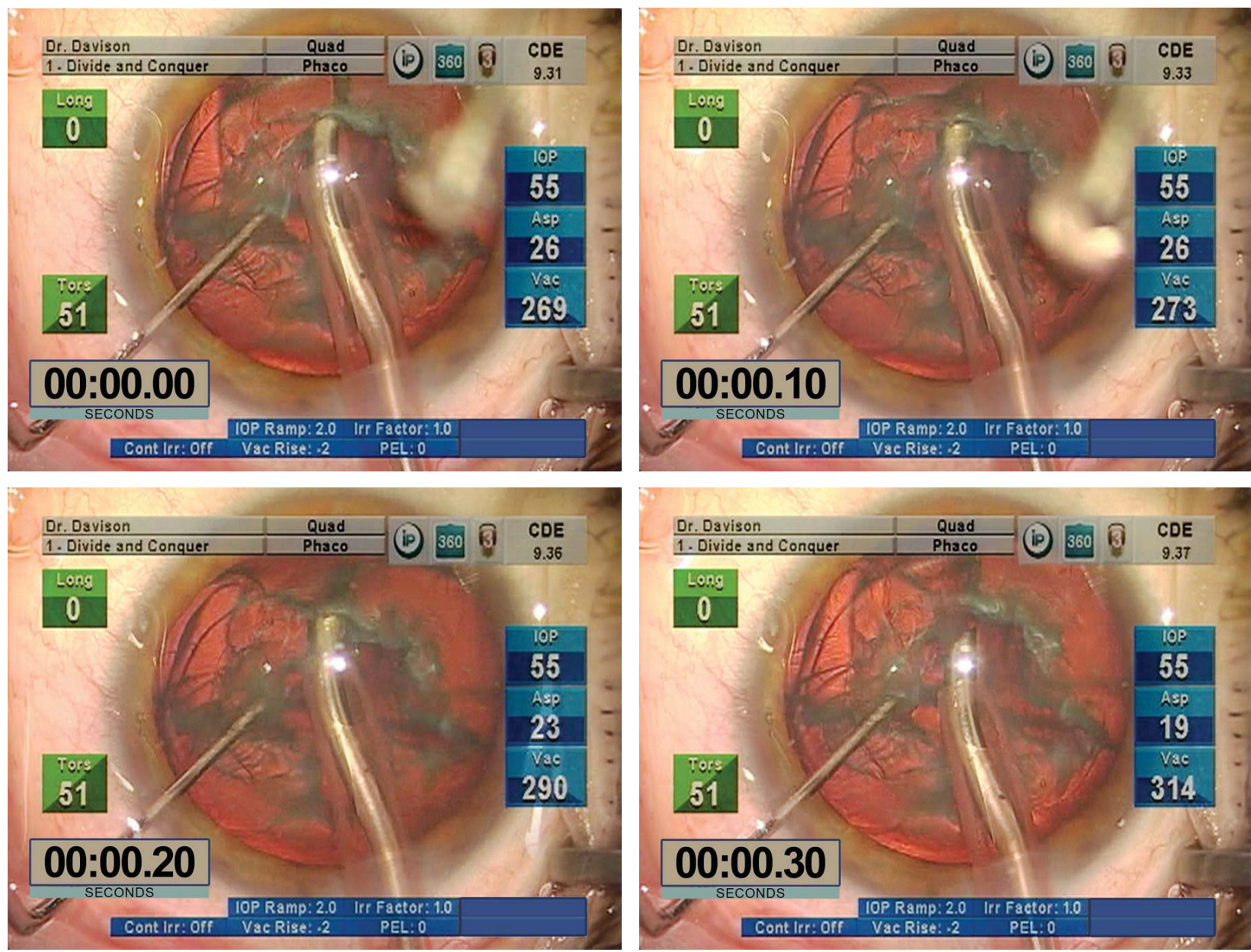

Figure 2 Video freeze frames from standard slow motion 30 frames/second video show the posterior capsule aspiration event.

Notes: At 0 seconds (top left) the tip aperture has just made contact with the edge of the quadrant. Torsional tip motion, vacuum, and aspiration flow are all active in foot position 3 and have already achieved high values. At 0.10 seconds (top right) nuclear material has started to be aspirated through the tip's aperture which is facing the peripheral posterior capsule but is well away from it. Early dimpling of the outer nuclear rim is occurring as the entire quadrant thickness is being drawn centrally by the vortex of relatively high vacuum and flow projecting from the tip aperture. At 0.20 seconds (bottom left) the full thickness of the quadrant has been aspirated into the aperture, and behind it a plug of cortex and posterior capsule as well. Nuclear material is still being drawn into the tip from the left and right. A faint outline of a punched out hole in the posterior capsule can be seen below the word "Phaco" on the display. The tip aperture is approximately $1.5 \mathrm{~mm}$ from the capsule. Maximum vacuum ( 400 $\mathrm{mmHg}$ ) has not been reached but aspiration flow and torsional values have remained high. At 0.30 seconds (bottom right) the hole in the cortical layer and posterior capsule is more easily seen. The remaining posterior capsule and surrounding cortex and peripheral nucleus have been released and have already been drawn back into their normal positions by zonular traction.

Abbreviations: IOP, intraocular pressure; Vac, vacuum.

effective, overall inefficiency was experienced because sometimes quadrant aspiration proceeded so slowly that we had to stop surgery, come out of the soft cataract menu, enter into the normal cataract menu, and then resume surgery. We selected between both menus until the next posterior capsule aspiration case occurred.

The second case was a 72 year old female operated in February 2015 after a total experience of 3,238 CENTURION cases. She had pseudoexfoliation and her lens was also relatively soft (LOCS III NO3.6 NC3.6) with grade 4 anterior and posterior nuclear clefting. We used the normal cataract menu for that case which resulted in a similar opening in the posterior capsule at the same point in surgery, but this time vitreous was also aspirated so an anterior vitrectomy was required. We saw a large nuclear fragment fall through the posterior capsular opening. The presence of it lying on the posterior pole was confirmed with indirect ophthalmoscopy, but we were still able to implant a posterior chamber IOL placed as in the first case. A pars plana vitrectomy was accomplished by one of our vitreoretinal surgeons 10 days after the patient's cataract surgery to remove the posterior nuclear fragment. Recovery was uneventful in that case as well. We had intended to use a 16.5 D Alcon SN60WF but instead used a 16.5 D Alcon MN60AC with a final refraction of $+0.50-0.50 \times 080$ and $20 / 20$ vision.

After that case we developed our current soft cataract setting parameter values and settings procedure step toolbar display, and have used it without incident for an additional 824 cases. Only preset terms are available in the CENTURION settings menu for inclusion as procedure step buttons 


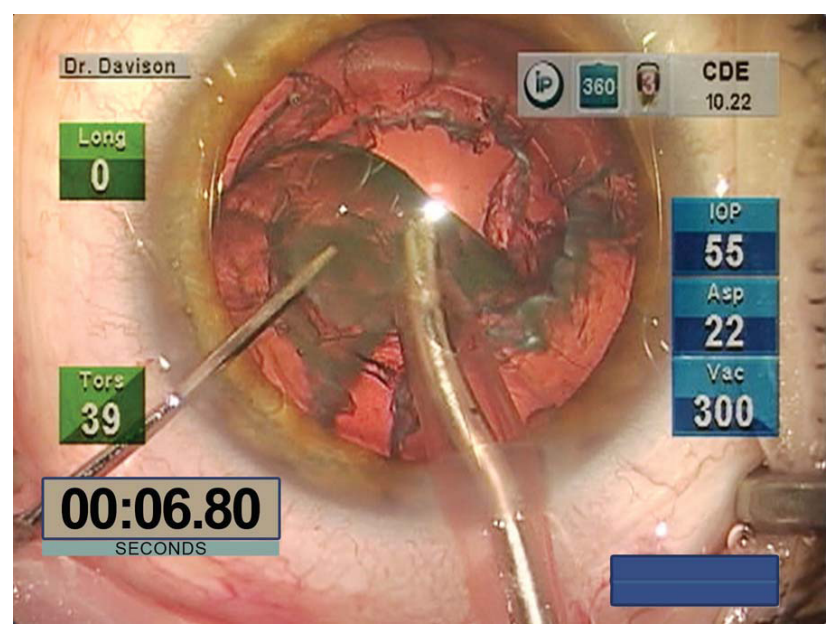

Figure 3 Video freeze frame of third quadrant removal.

Notes: At 6.8 seconds, the third quadrant has been acquired and phacoemulsification continues to be carefully accomplished while trying to stay away from the capsular defect. There was enough aqueous in front of the anterior hyaloid membrane so that it was insulated from being aspirated.

Abbreviations: Vac, vacuum; IOP, intraocular pressure.

into its procedure step toolbar, so we selected the Epinucleus Removal Setting designation for the soft cataract values. We inserted that setting button between Sculpt and Quadrant Removal buttons so that it would be instantaneously available to be used, skipped, or switched back and forth from as desired at any time without having to stop and change selection between two separate menus, namely the previously designated soft or normal prior to the start of surgery. The setting features simultaneous surgeon control of torsional amplitude, vacuum, and aspiration flow (Figure 4).

\section{Technique modifications for soft cataract phacoemulsification}

The surgical technique is most appreciated when applied to cataracts graded LOCS III NO and/or NC 3.6 or less but is employed in firmer lenses as well. Relatively narrow grooves are sculpted into the nucleus using the sculpt setting (Figure 5), sometimes after using an Akahoshi pre-chopper, Ambler Surgical, Exton, PA, USA to create four quadrants (LOCS III NO or NC 3.5 or less). The distance from the tip's aperture from the capsule, and the low vacuum and aspiration flow, make inadvertent capsule aspiration unlikely. Better visualization of the tip's cutting edge on the progressively thinner posterior nuclear plate is created by turning it into an oblique position with the aperture away from the surgeon (Figure 6A). Tearing of the posterior nuclear plate from peripheral to central is facilitated using the same tip orientation. The tip is then rotated approximately $90^{\circ}$ to achieve a different oblique orientation where the aperture is toward

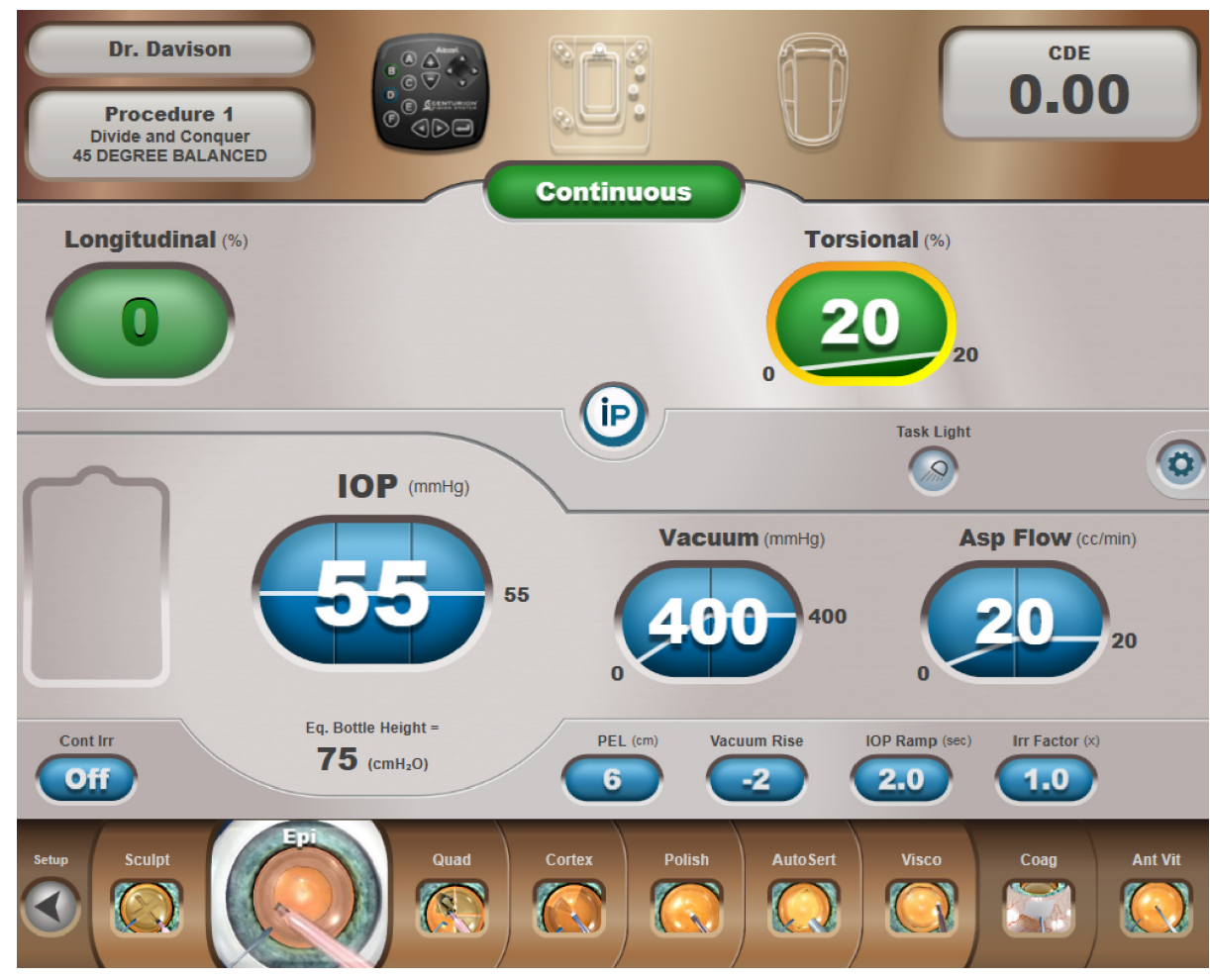

Figure 4 The machine panel display of the Epinucleus Removal Setting (Epinucleus Removal Step Button enlarged in the procedure step toolbar) shows linear control of vacuum and aspiration flow in foot position 2, which becomes fixed at their maximums in foot position 3 when linear control of continuous torsional amplitude becomes available. The iP feature is engaged but does not come into play in soft cataracts. The Epinucleus Removal Setting procedure step button is between the Sculpt and Quadrant Removal procedure step buttons in the procedure step toolbar along the bottom of the display.

Abbreviation: IOP, intraocular pressure. 


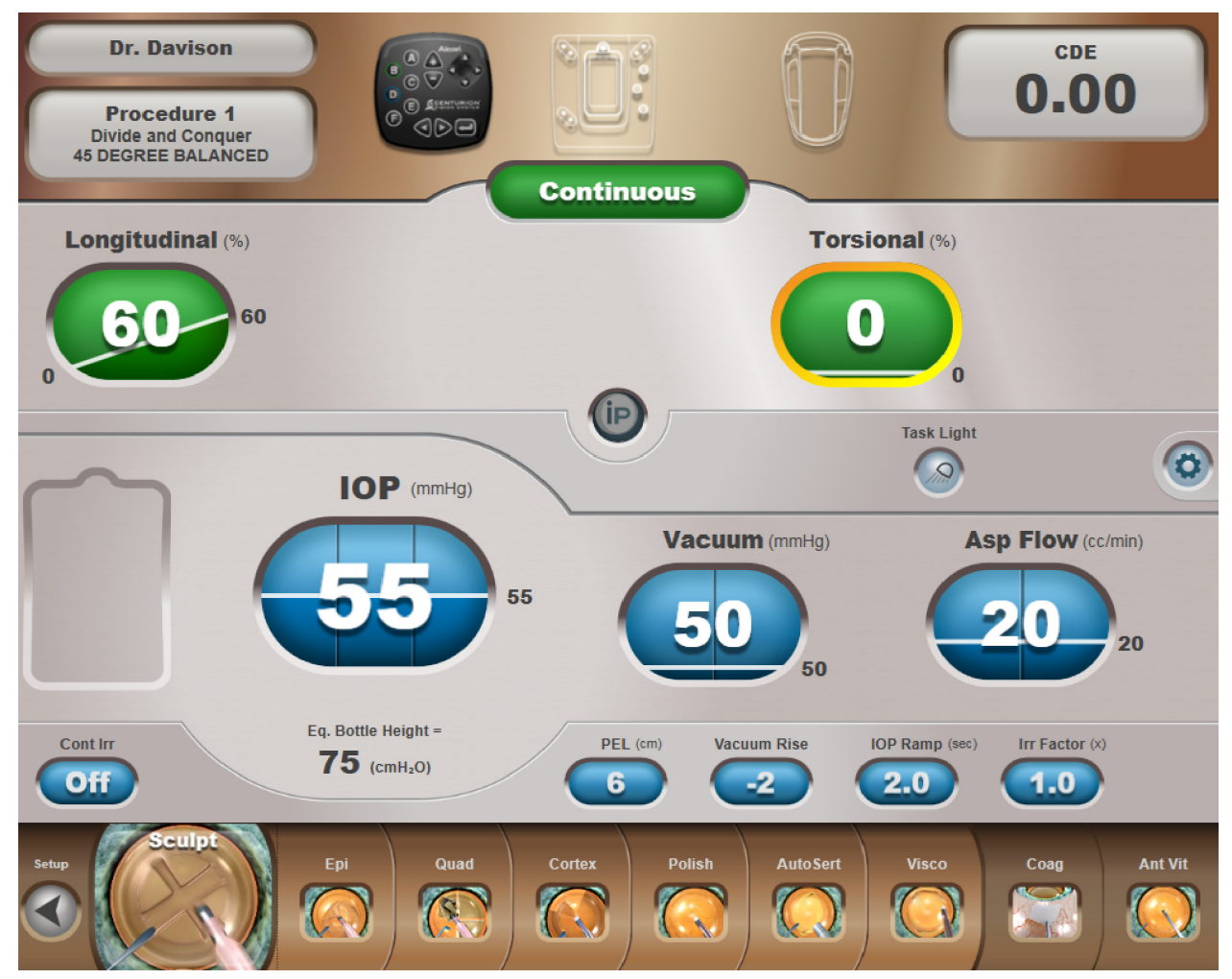

Figure 5 The machine screen display of the Sculpt Setting (Sculpt Procedure Step Button enlarged in the procedure step toolbar) shows low fixed values for vacuum and aspiration flow and linear control of continuous longitudinal tip motion.

Note: These parameters provide just enough vacuum and flow to aspirate the mix of nuclear dust in balanced salt solution.

Abbreviation: IOP, intraocular pressure.

the surgeon for deep nuclear shaving of quadrant corners to reduce nuclear bulk.

With the tip in the same orientation, the Epinucleus Removal Setting is engaged for quadrant acquisition and aspiration. Using a -2 vacuum rise time, linear control provides the ability for both vacuum and aspiration flow to be simultaneously increased to $400 \mathrm{mmHg}$ and $20 \mathrm{~mL} / \mathrm{min}$, respectively, throughout excursion through foot pedal position 2 . The machine attempts to hold these maximums as very minimal torsional tip motion is incrementally added with linear control from $0 \%-20 \%$ during travel through foot pedal position 3 . The quadrant acquisition and centralization process is stretched typically to $1.5-3$ seconds or even longer as desired. We had previously engaged the right corner of the quadrant but changed to acquire the left so that if that corner broke off, the aperture would face a relatively open area of BSS and the effects of vacuum and flow would not burst into the relatively closed and nonvisible space through a full thickness defect which might have been created in the right corner if it were to have been acquired and attempted to be drawn centrally. This change also allowed the cyclodialysis spatula to push the adjacent left quadrant away to open a space for the left corner of the first quadrant to rotate through as it was being pulled centrally (Figure 6).
The Quadrant Removal Setting is typically accessed for removal of the second and subsequent quadrants of relatively firm soft lenses (Figure 7). It may be activated even after the first quadrant of such lenses has been partially removed as centralization has been partially accomplished. The Intelligent Phaco feature is available in the quadrant removal setting but does not come into play in these softer cataracts.

For soft lenses where the posterior plate cannot be torn with a pre-chopper or by visco-fracture, ${ }^{1}$ the central and deep peripheral nucleus can be aspirated during sculpting and deeper nuclear thinning, thus creating the desired thin nuclear bowl. Using the Epinucleus Removal Setting, the peripheral nucleus can be progressively aspirated as it is rotated to provide new peripheral material for the tip to acquire and roll centrally. As the last peripheral section is drawn centrally, the attached posterior nucleus will usually follow with it and become centralized as well so aspiration of it can be completed away from the posterior capsule. ${ }^{2}$

We have found that we currently use the Epinucleus Removal Setting in some way in about one-half of our cases, usually unlocking the first quadrant but sometimes in later quadrants especially when centralizing the last. In the others, we skip through it and go directly to the Quadrant Removal Setting. 

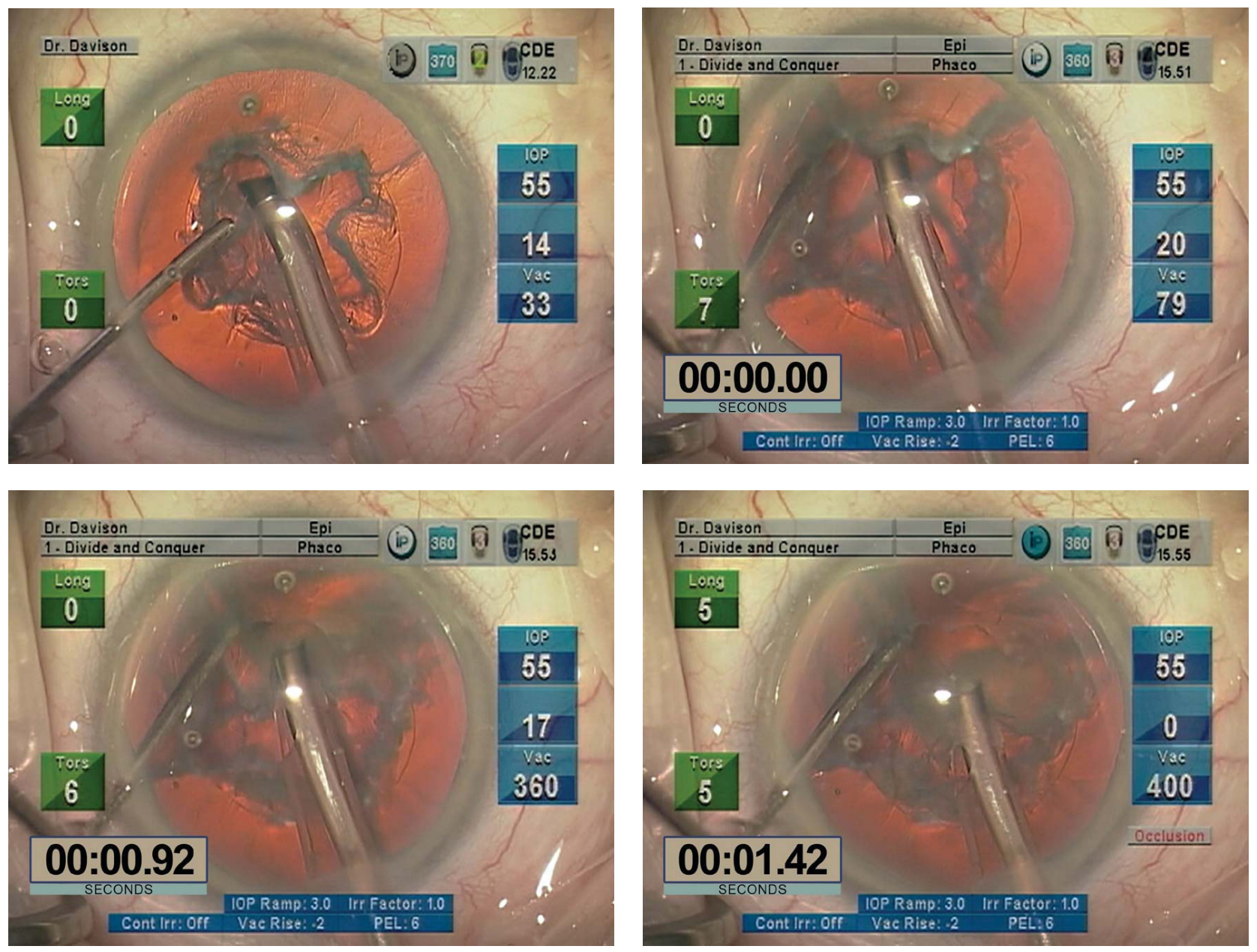

Figure 6 Soft cataract technique using the Epinucleus Removal Setting.

Notes: Top Left shows the Balanced Tip is turned slightly obliquely (aperture away from the surgeon) so that better visualization of its distal edge on the posterior nuclear plate is available. Deeper sculpting is more safely accomplished with this improved view. At 0 seconds (top right) the quadrants have been separated and their deeper corners shaved away. The aperture of the obliquely oriented tip (aperture towards the surgeon) is just becoming occluded as $7 \%$ torsional power is beginning to be used. With aspiration flow at $20 \mathrm{~mL} / \mathrm{min}$, vacuum is starting to build as the cyclodialysis spatula pushes the adjacent quadrant away, thus opening a space for the left edge of the first quadrant to roll through. If sudden occlusion break and excessive aspiration were to occur, the tip would break through the left edge of the quadrant and be in a free space and not break through to the posterior capsule. 0.92 seconds later (bottom left), the tip and the left edge of the attached quadrant are being drawn centrally by high vacuum while the tip burrows into the quadrant's substance. At 1.42 seconds, (bottom right) adhesion is maintained by low torsional motion which creates a continuous new surface to which the tip can bond with high vacuum. The tip aperture and the left half of the first quadrant have been centralized. Maximum vacuum has been achieved reducing flow to zero for this instant, but increasing torsional movement will break the occlusion allowing flow of nucleus into the aperture.

Abbreviations: IOP, intraocular pressure; Vac, vacuum.

\section{Discussion}

The Balanced Tip used with the CENTURION machine generates approximately twice the ultrasonic efficiency measured by cumulative dissipated energy $(\mathrm{CDE})$ as that experienced with the Partial Kelman Tip used with the INFINITI machine across all LOCS III cataract grades. Efficiency is a substantial advantage when operating hard cataracts because followability seems to be increased, material removal is faster, and the amount of movement of the tip within the incision as measured by $\mathrm{CDE}$ is substantially less that the amount at the tip aperture. The relative shaft movement reduction should help reduce the likelihood of thermal corneal injury in longer more difficult cases.
Another advantage of the platform is the reduction in "post-occlusion break surge". This surge is seen clinically as a sudden rush of fluid into the tip and a reduction in IOP immediately after an occluded tip becomes unoccluded. It occurs when the vacuum which is built up in the aspiration tubing and cassette during tip occlusion suddenly releases when the occlusion clears. Normal IOP is re-achieved when the infusion catches up to normalized aspiration. The amount of surge is typically measured in the laboratory by completely clamping the aspiration line, building vacuum to a certain level, and then fully releasing the clamp. The event is captured electronically and is defined as the area within the tracing of IOP fluctuation from clamp release to normal IOP over time. 

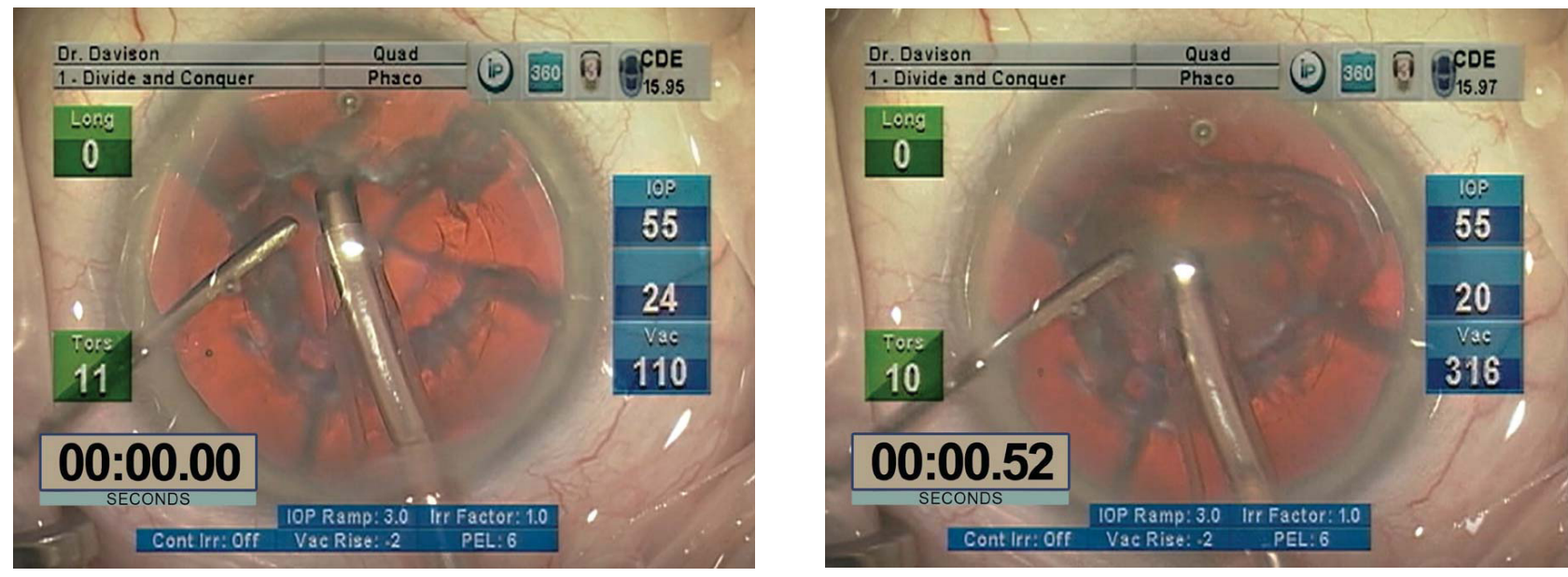

Figure 7 Removal of the second and subsequent quadrants of relatively firm soft lenses using the Quadrant Removal Setting.

Notes: With the timer set at 0 seconds (left) the second quadrant is about to be engaged using the quadrant removal setting. 0.52 seconds (right) later the tip aperture and second quadrant have been centralized. Nuclear material is being aspirated with high vacuum, intermediate aspiration flow, and minimal torsional tip movement.

Abbreviations: IOP, intraocular pressure; Vac, vacuum.

In one study of several phacoemulsification systems, using gravity fluidics with a $75 \mathrm{~cm}$ bottle height for each system, the INFINITI post-occlusion break surge area has been measured at $1.1 \pm 0.12 \mathrm{mmHg}$-seconds, while the CENTURION's was a fraction of it at $0.1 \pm 0.07 \mathrm{mmHg}$-seconds. ${ }^{5}$ The CENTURION with Active Fluidics (where the plastic BSS bag is compressed by a motor driven metal plate) and an equivalent target IOP of $55 \mathrm{mmHg}$ had a similarly reduced area at $0.2 \pm 0.06 \mathrm{mmHg}$-seconds. For comparison to other phacoemulsification systems in that study, the three Alcon systems used non-Aspiration Bypass System (ABS) tips whereas the tips used in our study have the normally included ABS shaft aperture. The reduction in surge in the CENTURION has been primarily achieved by decreasing compliance in the aspiration tubing and dual pump cassette and Active Fluidics software.

But even with advanced Active Fluidics, the transition to CENTURION/Balanced Tip technology required an empirical reduction in fluidic parameter values and ultrasonic energy delivery to achieve optimally controlled removal of softer nuclear quadrants. It would appear that this is primarily because the torsionally vibrating Balanced Tip is so effective at phacoemulsification. As seen in Figure 2 posterior capsule aspiration can happen even when the tip is well away from the capsule because of the extending vortex effects of vacuum and aspiration flow through the homogeneous gel-like lens material which is being very rapidly emulsified. It is not caused by anything as gross as complete post-occlusion break surge where all flow has been stopped and then is suddenly restored. It appears to be caused by a more subtle, dynamic, and nearly instantaneous process.
The physical characteristics of vitreous have been shown to cause nonuniform flow through a vitrector's aperture and lumen. ${ }^{14}$ Even greater nonuniform flow should be expected during the relatively highly energetic and erratic phacoemulsification process. Different shapes and viscosity materials are impacted or sheared by the tip's vibrating edge and are drawn into its lumen. Vacuum and flow variations should be caused by the continuum of soft gel to firm nuclear material, or by those materials as they are variably mixed with BSS as they tumble over and are drawn through the tip's aperture. As we further study the images in Figure 2, it is also interesting to contemplate that the posterior capsule, cortex, and soft relatively uniform peripheral nucleus are actually being deformed as they are pushed in that order toward and into the tip's aperture $1.5 \mathrm{~mm}$ away. In our video analysis case, the anterior hyaloid membrane and vitreous seemed to have been insulated by fluid between the membrane and posterior capsule so that they were not pushed toward the tip enough to be aspirated, but in our second case of vitreous loss they were. This "push" exists because of the relatively positive pressure within the globe created in part by the $55 \mathrm{mmHg}$ target IOP provided by the Active Fluidics hardware/ software and the negative pressure on the other side of the tip's aperture extending through its shaft and tubing created by the pump and cassette. At an equivalent bottle height of $75 \mathrm{~cm}$ above the eye, gravity-fed infusion would have done the same thing.

The trouble is these events can happen so quickly that surgeons have no time to react to them. Our video showed the entire posterior capsule aspiration event was 0.3 seconds (with capsule aspiration occurring in 0.2 seconds) while the reaction 
time from seeing an object to applying the brake in an automobile is on average 0.5 seconds. ${ }^{15}$ All of the vacuum and flow effects will be accelerated with increasing speed of the deformity and physical change of nuclear material by the extremely effective Balanced Tip. The greater the tip movement, the faster the physical changes in the material which is being emulsified, and the more apparent the flow changes will be.

The empirically derived parameters of the Epinucleus Removal Setting and its simultaneous linear control of vacuum, aspiration flow, and torsional tip motion slows the rate of acquisition and aspiration of softer nuclear material and gives the surgeon enough time and material over that time to centralize and further aspirate it. The control feedback sensation experienced by the surgeon is exactly the same as that experienced in irrigation/aspiration during cortex removal, so it might be thought of as "phaco-assisted I/A". If the name was available to choose in the machine's software, it could be labeled as "Soft Quadrant Removal" ahead of a "Normal Quadrant Removal". Or, since it is basically a reduction in the speed of acquisition and aspiration, it could be better labeled as "Slow Quadrant Removal" ahead of "Normal Quadrant Removal". Or it could be "First Gear" and "Second Gear" since it is analogous to using the first gear of an automobile to slowly pull away from the curb and then shift to second gear to build up speed and move along faster. Its intuitive nature can be seen through the scrub technicians' behavior. As they watch the video monitor, they can usually anticipate when I will want to shift gears and they have their finger on the remote control ready to shift on command.

Regardless of the setting name, we have instantaneous flexibility within the procedure step toolbar so that we can toggle back and forth instantly and almost automatically between these two speeds. We can shift between them at any point in the quadrant removal process, depending on the speed we want to achieve given the quadrant's texture, size, and proximity to the capsule. It can be especially useful for the manipulation and aspiration of the fourth quadrant when the protective physical buffering effect of the other quadrants is no longer available.

While crucial in soft nuclei, the Epinucleus Removal Setting can be used to slow down the pace of surgery in lenses of average firmness (LOCS III NO or NC 3.7 or greater) as well. It is especially useful in "unlocking" the first quadrant of average lenses. Many times, if the normal quadrant removal setting is used, the tip may just bite off corners of the nucleus and not allow enough time for the tip aperture to bond to the quadrant and roll it centrally. So, if that happens for the left corner of the first quadrant, the epinucleus setting can be used to more slowly access the left corner of the second. The introduction of the small amount of torsional movement creates a better vacuum adhesion by the tip and allows a slow burrowing into the nucleus. This happens because it continually creates a newly modified surface to adhere to as it vibrates. Once centralization and aspiration of the first portion of the first quadrant has been accomplished, the quadrant removal setting can be safely reengaged for removal of its remainder and subsequent quadrants as they will be no longer locked into position. Acquisition and centralization of quadrants in that setting typically takes about $1 / 2$ a second. As a caution, the shift to quadrant removal settings is instantaneous, so one must be ready for the instantaneously increased aspiration pace and amount of ultrasonic tip motion.

This report has a number of shortcomings. It is merely a clinical report of adaptation to technology, complication analysis, and further adaptation. It would be more scientific if it were a properly designed controlled prospective study between the applications of two parameter/technique groupings in similar populations with statistical analysis included. However, that clinical study could not be reasonably undertaken given the knowledge gained through our empirical evolution of parameters and techniques. Our study does importantly demonstrate the ability to reduce the pace of fragment acquisition and initial aspiration from 0.5 seconds to a much more manageable 1.5 seconds or longer, and the frequency of use of the soft cataract setting by this surgeon shows the efficiency and versatility of being able to shift between two speeds of phacoemulsification-assisted lens aspiration on the fly. The improvement in control and predictability has improved our confidence in the effectiveness and safety of the operation which we can deliver.

A laboratory study rather than clinical comparison may be more appropriate. Such a study has been accomplished comparing 20,23, and 25 gauge vitrectomy probes. ${ }^{16}$ Vitreoretinal surgeons need to be able to accurately and consistently attract and remove fibrous tissue sheets and bands without aspirating and cutting nearby or adjacent retinal tissue. The study measured the amount of artificial membrane deflection (the attraction distance) as a function of lumen diameters and variably applied vacuum and aspiration flow rates. It demonstrated that larger-gauge instruments operating with lower vacuum and higher flow attracted tissue at a greater distance than smaller instruments with higher vacuum and lower flow. Thus, the smaller probes would be more desirable as they would be less likely to inadvertently attract retinal tissue because they have a smaller distant "sphere of influence" than the larger 
ones. For phacoemulsification, some efficiencies of $0.9 \mathrm{~mm}$ diameter phacoemulsification tips compared to $1.1 \mathrm{~mm}$ tips have already been demonstrated. ${ }^{17}$ A study similar to the vitrectomy study could be accomplished using membrane deflection through a gel-simulating soft nuclear material as vacuum, flow, and phaco-tip movement, and maybe even tips smaller than $0.9 \mathrm{~mm}$ were studied.

\section{Conclusion}

While the setting parameter values and setting menu toolbar displays may be different than the CENTURION's with its Balanced Tip, the principles described for optimization, versatility, and flexibility should apply to other machines and tips as well. In any situation, preoperative grading of cataracts according to the LOCS III system can sort them into soft and normal and thus help dictate to the staff and surgeon which settings might be desirable to initiate removal of the first nuclear quadrant. Regardless of the initial plan, if the cataract seems soft during sculpting, a soft quadrant removal setting can be initially engaged to provide an increased margin of safety as the first nuclear quadrant is acquired and aspirated. For soft lenses, I recommend a slower speed of phacoemulsification which can be achieved by using simultaneous linear control of empirically derived parameters of aspiration flow, vacuum, and torsional tip motion. With this setting integrated into a machine's toolbar, immediate shifting between slow and normal speeds provides increased efficiency and safety during removal of any nuclear material.

\section{Disclosure}

The author is a consultant to Alcon but has no financial or proprietary interest in any material or method presented. The author reports no other conflicts of interest in this work.

\section{References}

1. Malavazzi GR, Nery RG. Visco-fracture technique for soft lens cataract removal. J Cataract Refract Surg. 2011;37:11-12.

Clinical Ophthalmology

\section{Publish your work in this journal}

Clinical Ophthalmology is an international, peer-reviewed journal covering all subspecialties within ophthalmology. Key topics include: Optometry; Visual science; Pharmacology and drug therapy in eye diseases; Basic Sciences; Primary and Secondary eye care; Patient Safety and Quality of Care Improvements. This journal is indexed on Submit your manuscript here: http://www.dovepress.com/clinical-ophthalmology-journal
2. Gomaa A, Liu C. Bowl-and-snail technique for soft cataract. J Cataract Refract Surg. 2011;37:8-10.

3. Packer M, Fishkind WJ, Fine IH, Seibel BS, Hoffman RS. The physics of phaco: a review. J Cataract Refract Surg. 2005;31:424-431.

4. Han YK, Miller KM. Comparison of vacuum rise time, vacuum limit accuracy, and occlusion break surge of 3 new phacoemulsification systems. J Cataract Refract Surg. 2009;35:1424-1429.

5. Sharif-Kashani P, Fanney D, Injev V. Comparison of occlusion break responses and vacuum rise times of phacoemulsification systems. $B M C$ Ophthalmol. 2014;14:96.

6. Zacharias J, Ohl CD. Fluid dynamics, cavitation, and tip-to-tissue interaction of longitudinal and torsional ultrasound modes during phacoemulsification. J Cataract Refract Surg. 2013;39:611-616.

7. Liu Y, Zeng M, Liu X, et al. Torsional mode versus conventional ultrasound mode phacoemulsification: randomized comparative clinical study. J Cataract Refract Surg. 2007;33:287-292.

8. Davison JA. Cumulative tip travel and implied followability of longitudinal torsional phacoemulsification. J Cataract Refract Surg. 2008; 34:986-990.

9. Ronquillo CC Jr, Zaugg B, Stagg B, et al. Determining optimal torsional ultrasound power for cataract surgery with automatic longitudinal pulses at maximum vacuum ex vivo. Am J Ophthalmol. 2014; 158:1262-1266.

10. Fishkind WJ. Management of the soft nucleus. In: Fishkind WJ, editor. Complications in Phacoemulsification: Avoidance, Recognition, and Management. New York, NY: Thieme; 2002:105-108.

11. Davison JA. Results of endocapsular phacofracture debulking of hard cataracts. Clinical Ophthalmol. 2015;9:1233-1238.

12. Payne M, Gerogescu D, Waite AN, Olson RJ. Phacoemulsification tip vacuum pressure: Comparison of 4 devices. J Cataract Refract Surg. 2006;32: 1374-1377.

13. Davison JA, Chylack LT Jr. Clinical application of the Lens Opacities Classification System III in the performance of phacoemulsification. $J$ Cataract Refract Surg. 2003;29:138-145.

14. Magalhaes O Jr, Chong L, DeBoer C, et al. Vitreous dynamics: vitreous flow analysis in 20-, 23-, and 25-gauge cutters. Retina. 2008; 28:236-241.

15. Public Policy Center, The University of Iowa. Rear End Crash Avoidance System (RECAS) Algorithms and Altering Strategies: Effects of Adaptive Cruise Control and Alert Modality on Driver Performance. Final report. University of Iowa; 2006.

16. Dugel PU, Zhou J, Abulon DK, Buboltz DC. Tissue attraction associated with 20-gauge, 23-gauge, and enhanced 25-gauge dual-pneumatic vitrectomy probes. Retina. 2012;32:1761-1766.

17. Davison JA. Performance comparison of the Alcon Legacy 20000 $1.1 \mathrm{~mm}$ TurboSonics and $0.9 \mathrm{~mm}$ MicroTip. J Cataract Refract Surg. 1999;25:1382-1385.

\section{Dovepress}

PubMed Central and CAS, and is the official journal of The Society of Clinical Ophthalmology (SCO). The manuscript management system is completely online and includes a very quick and fair peer-review system, which is all easy to use. Visit http://www.dovepress.com/ testimonials.php to read real quotes from published authors. 